\title{
Conductive Composite Films Prepared Using Undoped Polyaniline and Poly(methyl methacrylate)
}

\author{
Meral KaraKişLa, Emine Erdem, ${ }^{*}$ and Mehmet SAÇAK ${ }^{\dagger}$ \\ Department of Chemistry, Faculty of Science, Ankara University, 06100 Tandoğan, Ankara, Turkey \\ * Department of Chemistry, Faculty of Education, Hacettepe University, 06532 Beytepe, Ankara, Turkey
}

(Received May 15, 2003; Accepted September 4, 2003)

\begin{abstract}
In this study, undoped polyaniline-poly(methyl methacrylate) films were cast from solutions containing different amounts of soluble part of undoped polyaniline and poly(methyl methacrylate) in dimethylformamide. The films contained undoped polyaniline up to $60 \%$ since the solubility of undoped polyaniline in dimethylformamide was $1.14 \mathrm{~g} / 100 \mathrm{~mL}$. The conductivity of undoped polyaniline-poly(methyl methacrylate) films were investigated after being redoped in some acids such as oxalic, nitric, and hydrochloric acids. When the films doped with hydrochloric acid was kept in air, the conductivity decreased gradually by $32 \%$. Their temperature sensor behavior was examined in the range of -15 to $120^{\circ} \mathrm{C}$. The characterization of films was performed by conductivity measurement, FT-IR, UV, and TGA techniques.
\end{abstract}

KEY WORDS Polyaniline / Conductive Film / Poly(methyl methacrylate) / Composite /

Polyaniline (PAn) is one of the intrinsically conducting polymers (ICPs). Its doped form is insoluble in common organic solvents and decomposes before it melts. ${ }^{1}$ That is why it is not always possible to cast into different forms. The chemical polymerization gives a powdered PAn. ${ }^{2-4}$ The electrochemical polymerization of aniline gives a film on the electrode with very poor mechanical properties. The PAn film formed on the electrode surface after the electrolysis can not be peeled off in a freestanding form..$^{-7}$

There are various methods to improve the mechanical properties of polyaniline. One of them is preparation of the composites or blends of polyaniline with an insulating polymer having better mechanical properties. $^{8-13}$ The preparation of conductive films can be carried out by both the electrochemical and chemical ways. In the electrochemical method the surface of the electrode is covered with an insulating polymer in advance and then this electrode is used as a working electrode in the electrochemical oxidation of aniline. ${ }^{14-16}$ In the chemical method the polymerization of aniline is carried out in a solution of an insulating polymer and the resulting mixture is cast into films on a smooth surface such as glass in order to obtain polyanilineinsulating polymer conducting film. ${ }^{8,12}$ In an alternative method, conductive PAn is directly mixed with a solution of an insulating polymer and films are cast from the solution. However, in this method it is not possible to control the homogeneity of the conducting PAn particles in the film. ${ }^{10,17,18}$

PAn doped with specific dopant ions can be soluble to a certain extent in some organic solvents such as dimethylsulphoxide, dimethylformamide and $N$-methylpyrrolidone. ${ }^{19-21}$ It is also reported that the solubility of undoped PAn is higher in these solvents than that of the conductive form. ${ }^{22-24}$

This study deals with a new method of preparation of PAn-insulating polymer films. In this method, soluble parts of undoped PAn in DMF and poly(methyl methacrylate) were mixed together in DMF and the mixture was cast into undoped films. Undoped films obtained by this way were doped with various acids in order to convert them into conductive PAn-poly(methyl methacrylate) films. These films were characterized by means of conductivity measurement, FT-IR, UV-vis, and TGA techniques.

\section{EXPERIMENTAL}

\section{Materials}

Aniline was distilled under vacuum. Poly(methyl methacrylate) (PMMA) with a viscosity average molecular weight of 150000 was synthesized by solution polymerization using benzoyl peroxide. Other chemicals were used as purchased from Merck Co.

\section{Procedures}

Aniline was polymerized in $1.0 \mathrm{M}$ oxalic acid solution with $\mathrm{K}_{2} \mathrm{Cr}_{2} \mathrm{O}_{7}$ at $25^{\circ} \mathrm{C}$. The aniline/oxidant mole ratio was $6 / 1$. The mixture was allowed to react for $24 \mathrm{~h}$. PAn doped with oxalic acid precipitated in the final stage of the polymerization was filtered and the polymer was washed with water. The conductive PAn sample was treated with $3.0 \mathrm{M} \mathrm{NH}_{3}$ solution for $8 \mathrm{~h}$.

${ }_{\dagger}^{\dagger}$ To whom correspondence should be addressed (E-mail: sacak@science.ankara.edu.tr). 
PAn being freed from the dopant was filtered and washed with excess water and dried.

The undoped PAn sample was added to excess DMF and stirred at room temperature for $4 \mathrm{~h}$. The insoluble part of the PAn was filtered. The filtrate was kept in air to remove the solvent, which left undoped PAn soluble in DMF. These parts of undoped PAn were used to obtain undoped PAn-PMMA films containing undoped PAn at different ratios in DMF.

The films were prepared by mixing the solutions containing presribed amounts of the undoped PAn and the PMMA $(0.5 \%)$ in DMF. The mixtures were dropped on glass plates $\left(1.5 \times 1.5 \mathrm{~cm}^{2}\right)$ and dried at room temperature for $24 \mathrm{~h}$ in ambient atmosphere and then in vacuum at $50{ }^{\circ} \mathrm{C}$ for $12 \mathrm{~h}$. The films with different thickness were obtained by changing the amount of the solution put on the glass plate. The thickness of the films was determined by the use of a micrometer with sensitivity of $0.01 \mathrm{~mm}$ by taking average of the thickness at 5 different points.

\section{Measurements}

The conductivity of the samples was measured by the use of four-probe technique. FT-IR spectra of the doped and undoped PAn-PMMA films and pure polymers were taken with $\mathrm{KBr}$ pellets by using a Matsoon 1000 FT-IR spectrometer. UV-vis spectra were obtained by the use of Shimadzu 160 A spectrophotometer. Thermogravimetric analysis was carried out by using a Linseis Model thermal analyzer under He atmosphere with a flow rate of $10 \mathrm{~mL} \mathrm{~min}^{-1}$ at a heating rate of $10^{\circ} \mathrm{C} \mathrm{min}{ }^{-1}$.

Temperature dependence of the resistance was measured in a temperature range of -15 to $+120^{\circ} \mathrm{C}$. The film attached to a copper wire using a conducting $\mathrm{Ag}$ paint was placed in a temperature-controlled chamber with a heating rate of $3^{\circ} \mathrm{C} \mathrm{min}^{-1}$. The resistance of the conducting PAn-PMMA film was recorded as a function of temperature.

\section{RESULTS AND DISCUSSION}

\section{Conductivity of Composite Films}

PMMA, which is soluble in DMF, was used as the insulating polymer since undoped PAn is partly soluble in DMF. The solubilities of oxalic acid in the doped and undoped PAn are $0.008 \mathrm{~g} / 100 \mathrm{~mL}$ and $1.14 \mathrm{~g} / 100 \mathrm{~mL}$, respectively. The films containing the undoped PAn up to $60 \%$ were prepared due to limitation of the solubility of undoped PAn in DMF.

The PAn-PMMA film containing 60\% undoped PAn was easily peeled off the glass surface. The comparison of this film with the ones containing lower amount of

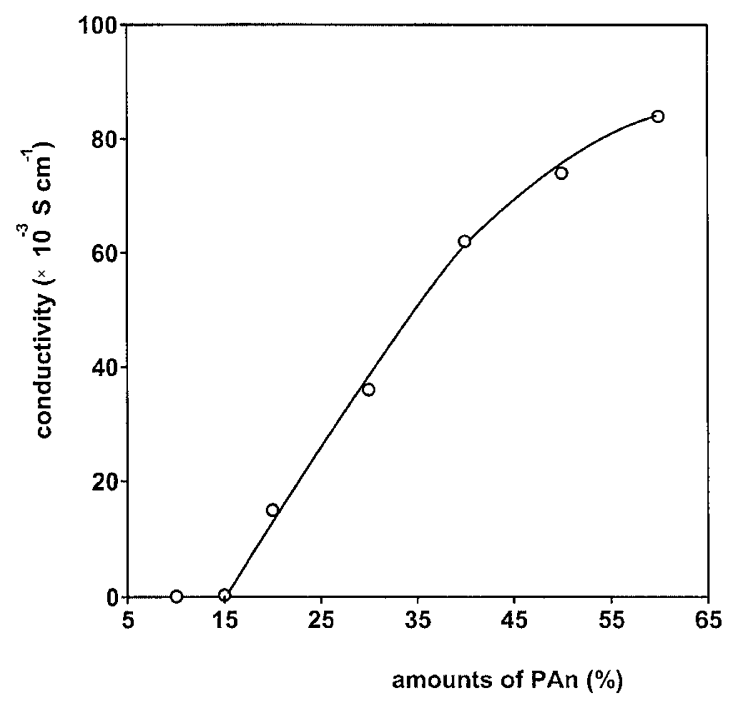

Figure 1. Effect of PAn content on conductivity at $25^{\circ} \mathrm{C}$ for PAn-PMMA films doped with $2.0 \mathrm{M} \mathrm{HCl}$, for $1 \mathrm{~h}$. Film thickness was $0.07 \mathrm{~mm}$.

undoped PAn showed no significant difference in durability.

The undoped PAn-PMMA films were redoped by keeping them in $1.0 \mathrm{M}$ aqueous oxalic acid for an hour. Dark blue color of undoped PAn-PMMA films changed into dark green, which is characteristic of conductive PAn. The conductivity of the doped film containing $60 \%$ PAn was found to be $1.3 \times 10^{-5} \mathrm{~S} \mathrm{~cm}^{-1}$. The conductivity values of films having undoped PAn lower than $60 \%$ were out of measurement limit $\left(10^{-6}\right.$ $\mathrm{S} \mathrm{cm} \mathrm{cm}^{-1}$ ) in our laboratory conditions. Similarly the doping process of the film containing $60 \%$ undoped PAn with $\mathrm{HNO}_{3}$ resulted in a conductivity value of $5.6 \times 10^{-5} \mathrm{~S} \mathrm{~cm}^{-1}$.

High conductivity values were observed by doping of films in $2.0 \mathrm{M} \mathrm{HCl}$. As seen from Figure 1 the films containing $10-60 \%$ undoped PAn after the doping treatment gave conductivity values in the range of $0.05 \times 10^{-3}$ to $8.5 \times 10^{-3} \mathrm{~S} \mathrm{~cm}^{-1}$. The conductivity of the PAn-PMMA film increased with the amount of PAn they contain. The conductivity of pure PAn doped in $2 \mathrm{M} \mathrm{HCl}$ was $0.25 \mathrm{~S} \mathrm{~cm}^{-1}$. The conductive form of PAn-PMMA film was redoped in $3.0 \mathrm{M} \mathrm{NaOH}$.

Table I shows the dependence of conductivity of films on $\mathrm{HCl}$ concentration employed in doping process. The experiments were carried out on films containing $60 \%$ undoped PAn. It was found that the conductivity of the film increased with the dopant concentration and then leveled off as mentioned in numerous studies. ${ }^{25,26}$

The conductivity of the films is affected by the diffusion capability of the dopant ions into the film. Therefore waiting period in $\mathrm{HCl}$ and the thickness of the film is of great importance for homogenous distribution of 
Table I. Effect of concentration of $\mathrm{HCl}$ used as dopant on the conductivity of $\mathrm{HCl}$ doped PAn-PMMA film containing 60\% undoped PAn

\begin{tabular}{cc}
\hline $\begin{array}{c}{[\mathrm{HCl}]} \\
\left(\mathrm{mol} \mathrm{L}^{-1}\right)\end{array}$ & $\begin{array}{c}\text { Conductivity } \\
\left(10^{-3} \mathrm{~S} \mathrm{~cm}^{-1}\right)\end{array}$ \\
\hline 0.5 & 0.9 \\
1.0 & 3.7 \\
1.5 & 5.8 \\
2.0 & 8.5 \\
2.5 & 8.2 \\
3.0 & 8.7 \\
\hline
\end{tabular}

Thickness, $0.07 \mathrm{~mm}$; temperature, $25^{\circ} \mathrm{C}$; doping period, $1 \mathrm{~h}$.

Table II. Effects of film thickness and doping period in $2.0 \mathrm{M}$ $\mathrm{HCl}$ on the conductivity of PAn-PMMA film containing $60 \%$ PAn

\begin{tabular}{ccccc}
\hline \multirow{2}{*}{ Time (min) } & \multicolumn{4}{c}{ Conductivity $\left(10^{-3} \mathrm{~S} \mathrm{~cm}^{-1}\right)$} \\
& $0.05 \mathrm{~mm}$ & $0.07 \mathrm{~mm}$ & $0.09 \mathrm{~mm}$ & $0.12 \mathrm{~mm}$ \\
\hline 5 & 0.45 & 2.2 & 2.6 & 1.7 \\
10 & 0.56 & 3.6 & 3.5 & 2.8 \\
30 & 0.62 & 6.3 & 5.2 & 4.5 \\
60 & 0.96 & 8.5 & 6.8 & 6.0 \\
120 & 1.02 & 8.8 & 8.1 & 7.5 \\
\hline
\end{tabular}

Temperature, $25^{\circ} \mathrm{C} ;[\mathrm{HCl}], 2.0 \mathrm{M}$.

the dopant ions. In order to investigate these effects the films containing $60 \%$ undoped PAn with varying thickness were kept in $2.0 \mathrm{M} \mathrm{HCl}$ for different periods. The conductivities of samples were given in Table II.

The film reached to a certain conductivity value within five minutes and the conductivity increased with the doping period. In the films with thickness of 0.05 and $0.07 \mathrm{~mm}$ the conductivity reached equilibrium after one hour. The doping period of two hours in films with thickness of 0.09 and $0.12 \mathrm{~mm}$ gave higher conductivity values than that those obtained with a doping period of one hour. Therefore even a period of two hours is not sufficient in these films for attaining equilibrium distribution of the ions in the films.

Figure 2 shows the effect of waiting period in air upon the conductivity of film. This experiment was carried out using PAn-PMMA film doped in $2.0 \mathrm{M} \mathrm{HCl}$ for one hour. The conductivity of the film was decreased with time and remained constant at $5.8 \times 10^{-3} \mathrm{~S} \mathrm{~cm}^{-1}$ after $6 \mathrm{~d}$. The decrease in conductivity in this period was approximately $32 \%$.

\section{FT-IR Spectra}

Figure 3 shows the FT-IR spectra of (a) PMMA, (b) conductive PAn, (c) undoped, and (d) conductive PAnPMMA films. Among these films, conductive films were prepared by doping with $\mathrm{HCl}$. The $\mathrm{C}=\mathrm{O}$ band observed at $1722 \mathrm{~cm}^{-1}$ in PMMA and the bands observed at 1486 and $1569 \mathrm{~cm}^{-1}$ belonging to the $\mathrm{C}-\mathrm{N}$ and $\mathrm{C}=\mathrm{N}$ bands of benzenoid and quinoid units ${ }^{27}$ in doped PAn

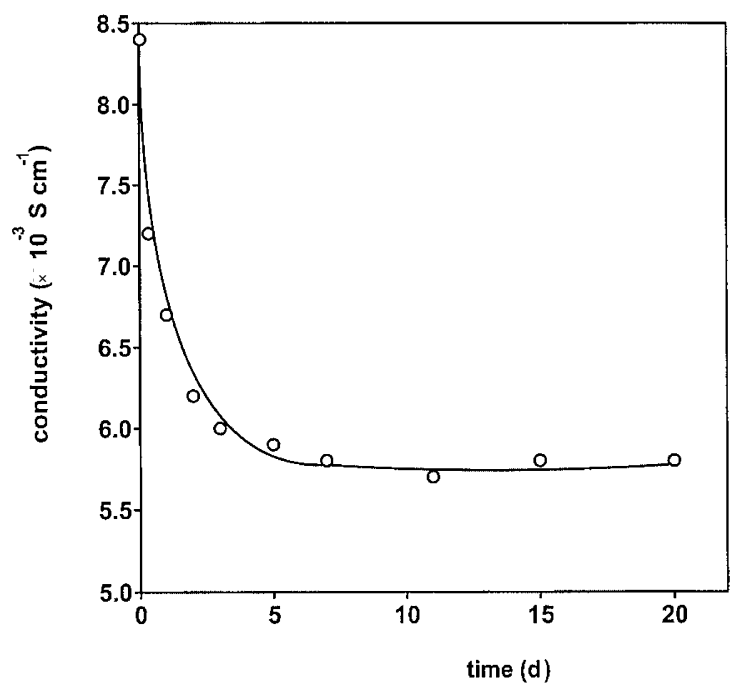

Figure 2. Effect of waiting period in air upon the conductivity of PAn-PMMA films doped with $2.0 \mathrm{M} \mathrm{HCl}$. The thickness of film was $0.07 \mathrm{~mm}$.

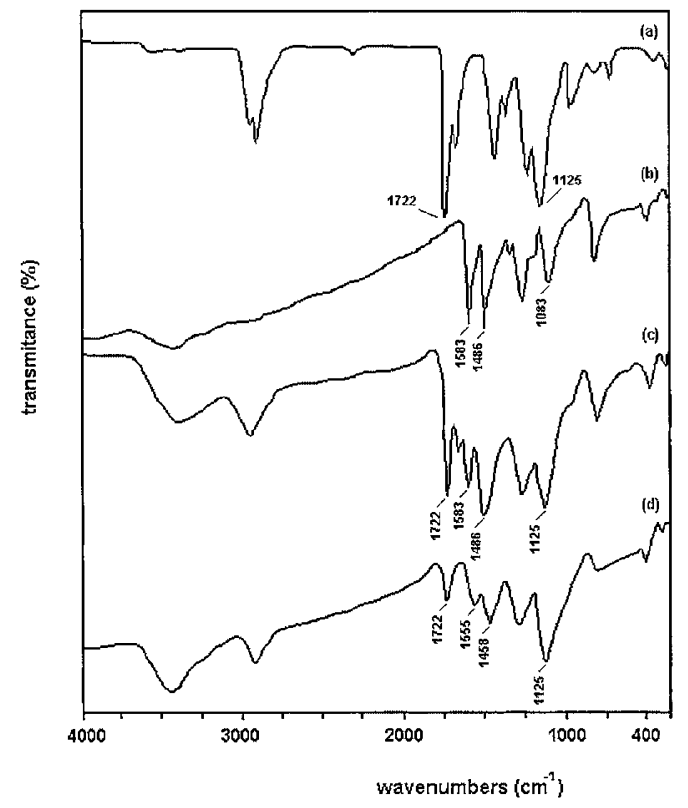

Figure 3. FT-IR spectra of (a) PMMA, (b) doped PAn, (c) undoped PAn-PMMA film, and (d) doped PAn-PMMA film.

are clearly seen in the doped PAn-PMMA film.

The bands observed at 1500 and $1583 \mathrm{~cm}^{-1}$ in the undoped PAn-PMMA film (spectrum c) shift to 1486 and $1569 \mathrm{~cm}^{-1}$ in the conductive film (spectrum d), respectively. This shift corresponds to benzenoid-quinoid ring transitions. ${ }^{28}$ Also the intensity of peak at $1150 \mathrm{~cm}^{-1}$, which is characteristic to conductive PAn (spectrum b), decreases in the undoped PAn-PMMA film (c) and appears in an intensive and broader fashion in the conductive PAn-PMMA film (d). This conductivity band corresponds to charge delocalization on the PAn chains. ${ }^{29}$

\section{$U V$-vis Spectra}

The UV-vis spectra taken by the use of the undoped 


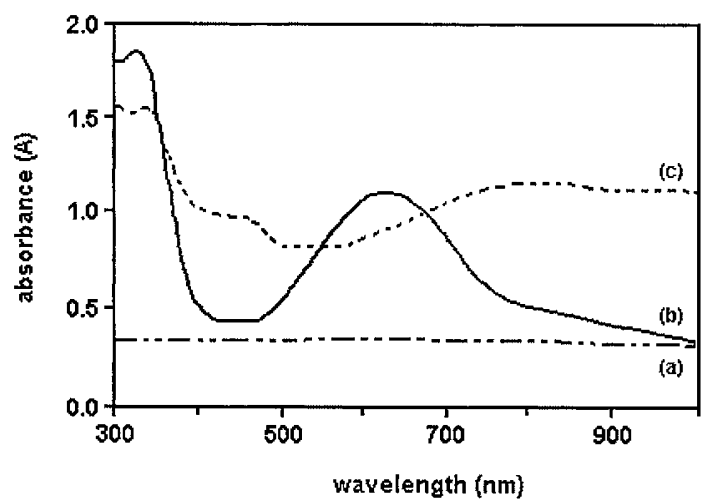

Figure 4. UV-vis spectra of (a) PMMA film, (b) undoped PAnPMMA film, and (c) PAn-PMMA film doped with $2.0 \mathrm{M} \mathrm{HCl}$.

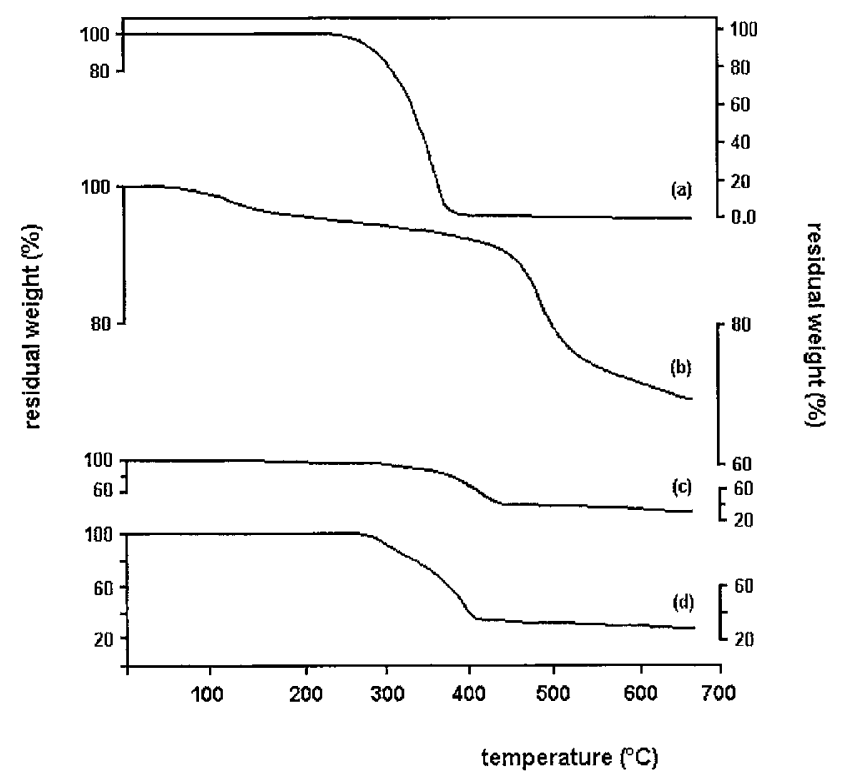

Figure 5. TGA thermograms of (a) PMMA, (b) doped PAn, (c) undoped PAn-PMMA film, and (d) PAn-PMMA doped with $2.0 \mathrm{M}$ $\mathrm{HCl}$.

PAn-PMMA film peeled off from the glass surface are shown in Figure 4. The band at $630 \mathrm{~nm}$ observed in the undoped PAn-PMMA film (b) is due to a $\pi-\pi^{*}$ transition of quinone-imine groups. ${ }^{30}$ This band disappears in the conductive PAn-PMMA film (c) and two new bands appear at 456 and $800 \mathrm{~nm}$ which are related to the $\pi-\pi^{*}$ transitions of quinone-iminium ions and the presence of localized cation radicals, respectively. ${ }^{30-32}$

\section{$T G A$}

Figure 5 shows the thermograms of PMMA, doped PAn, undoped and doped PAn-PMMA films taken in a temperature range of $20^{\circ} \mathrm{C}$ to $680^{\circ} \mathrm{C}$. It is observed that the weight loss of pure PMMA (a) starts at $270^{\circ} \mathrm{C}$ and looses all its weight at $680^{\circ} \mathrm{C}$. The first weight loss of doped PAn gives range of $100-300^{\circ} \mathrm{C}$ due to removal of water and dopant molecules. ${ }^{26}$ The second weight loss starting at $300^{\circ} \mathrm{C}$ corresponds to the degradation of the PAn chain and it looses $58 \%$ of its total weight

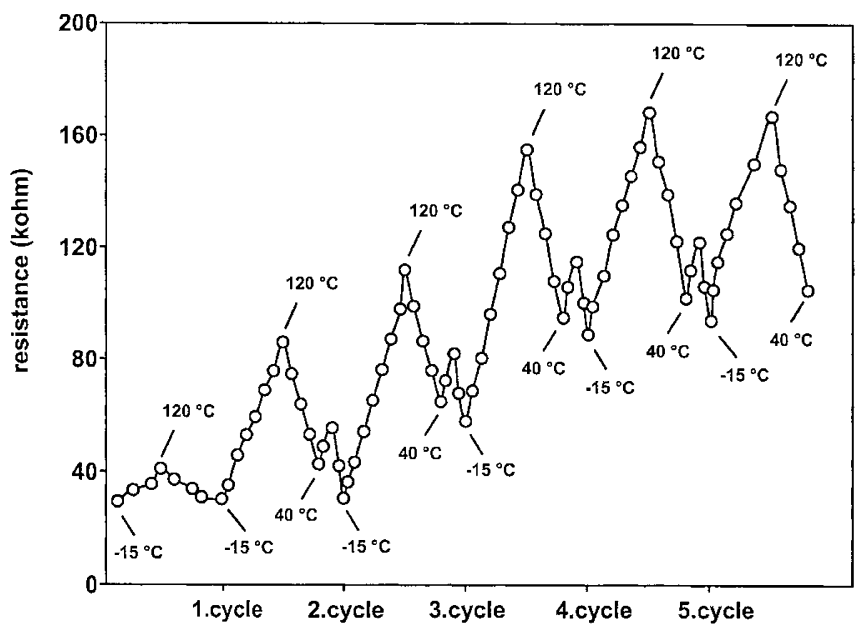

Figure 6. Temperature dependence of the resistance of conductive PAn-PMMA film in the range from $-15^{\circ} \mathrm{C}$ to $120^{\circ} \mathrm{C}$.

at $680^{\circ} \mathrm{C}(\mathrm{b})$. On the other hand, the weight losses of the undoped PAn-PMMA and doped PAn-PMMA films are $76 \%$ and $85 \%$ at $680^{\circ} \mathrm{C}$, respectively (c and d). These data indicate that both the undoped and doped PAn-PMMA films have higher thermal stability than PMMA and lower thermal stability than doped PAn.

\section{Thermal Sensor Properties}

Figure 6 shows the change in the resistance of the doped PAn-PMMA film with the temperature. The curve in Figure 6 was obtained by heating the film from -15 to $+120^{\circ} \mathrm{C}$ and then cooling down to $-15^{\circ} \mathrm{C}$. The resistance of doped PAn-PMMA film increased during the heating process. However the resistance decreased in the range from $120^{\circ} \mathrm{C}$ to $40^{\circ} \mathrm{C}$, increased in the range from $40^{\circ} \mathrm{C}$ to $10^{\circ} \mathrm{C}$ and decreased again below $10^{\circ} \mathrm{C}$ during the cooling process. The results of five heating and cooling cycles revealed that the doped PAn-PMMA film gave a reversible response against the temperature.

\section{CONCLUSION}

In this study, conductive PAn-PMMA films having excellent mechanical strength were prepared using a new method. Firstly, ternary solutions of undoped PAn and PMMA in DMF were cast into films and then these films were doped with acids such as oxalic acid, $\mathrm{HNO}_{3}$ and $\mathrm{HCl}$ to obtain conductive PAn-PMMA films. Due to the soluble part of undoped PAn was used these films were highly homogenous. The dopants were removed to be remarkable by treating with $\mathrm{NaOH}$. Films having the highest conductivity were obtained by doping with $\mathrm{HCl}$. It was found that undoped and doped PAnPMMA films have higher thermal stability than PMMA and lower thermal stability than doped PAn. 
Acknowledgment. The authors would like to thank Ankara University, Research Fund for the support of this work.

\section{REFERENCES}

1. T. Nakajima and T. Kawagoe, Synth. Met., 28, C6294 (1989).

2. J. C. Chiang and A. G. MacDiarmid, Synth. Met., 13, 193 (1986).

3. A. Pron, F. Genoud, C. Menardo, and M. Nechtschein, Synth. Met., 24, 193 (1988).

4. S. P. Armes and J. F. Miller, Synth. Met., 22, 385 (1988).

5. P. M. McManus, S. C. Yang, and R. J. Cashman, J. Chem. Soc., Chem. Commun., 1556 (1986).

6. J. C. Lacroix, K. K. Kanazawa, and A. Diaz, J. Electrochem. Soc., 136, 1308 (1989).

7. A. Watanabe, K. Mori, Y. Iwaski, S. Murakawi, and Y. Nakamura, J. Polym. Sci., Polym. Chem. Ed., 27, 4431 (1989).

8. N. V. Bhat and N. V. Joshi, J. Appl. Polym. Sci., 50, 1423 (1993).

9. W. A. Gazotti JR, R. Foez, and M. A. De Paoli, Eur. Polym. J., 35, 35 (1999).

10. M. De Paoli, E. R. Duek, and M. A. Rodrigues, Synth. Met., 41-43, 973 (1991).

11. C. K. Subramaniam, A. B. Kaiser, P. W. Gilberd, and B. Wessling, J. Polym. Sci., Part B: Polym. Phys., 31, 1425 (1993).

12. S. Yang and E. Ruckenstein, Synth. Met., 59, 1 (1993).

13. H. Morgan, P. J. S. Foot, and N. W. Brooks, J. Mater. Sci., 36, 5369 (2001).
14. S. A. Chen and W. G. Fang, Macromolecules, 24, 1242 (1991).

15. G. Bidan and B. Ehui, J. Chem. Soc., Chem. Commun., 1568 (1989).

16. M. Karakişla, M. Saçak, and U. Akbulut, J. Appl. Polym. Sci., 59, 1347 (1996).

17. B. Wessling and H. Volk, Synth. Met., 18, 671 (1987).

18. R. C. Patil, T. Aoyanagi, M. Nakayama, K. Ogura, J. Appl. Polym. Sci., 81, 2661 (2001).

19. S. Li, Y. Cao, and Z. Xue, Synth. Met., 20, 141 (1987).

20. Y. Cao, P. Smith, and A. J. Heeger, Synth. Met., 48, 91 (1992).

21. E. Erdem, M. Saçak, and M. Karakişla, Polym. Int., 39, 153 (1996).

22. Y. Wei, Y. Sun, and X. Tang, J. Phys. Chem., 93, 4878 (1989).

23. J. Yue and A. J. Epstein, J. Am. Chem. Soc., 112, 2800 (1990).

24. M. Angelopoulos, C. E. Asturier, S. P. Ermer, E. Ray, E. M. Scherr, A. G. MacDiarmid, M. A. Akhtar, Z. Kiss, and A. J. Epstein, Mol. Cryst. Liq. Cryst., 160, 151 (1988).

25. Y. Roichman, G. I. Titelman, M. S. Silverstein, A. Siegmann, and M. Narkis, Synth. Met., 98, 201 (1999).

26. E. Ruckenstein and S. Yang, Synth. Met., 53, 283 (1993).

27. H. Yan and N. Toshima, Synth. Met., 69, 151 (1995).

28. D. C. Trivedi and S. K. Dhawan, Synth. Met., 58, 309 (1993).

29. S. Stafstrom and B. Sjorgren, Synth. Met., 16, 31 (1986).

30. M. Inoue, R. E. Navarro, and M. B. Inoue, Synth. Met., 30, 199 (1989).

31. E. M. Genies and M. Lapkowski, J. Electroanal. Chem., 220, 67 (1987).

32. J. Stejskal, P. Kratochvil, and R. Adhakrishman, Synth. Met., 61, 225 (1993). 